\title{
ORTHOGONAL POLYNOMIALS AND RELATED SPECIAL FUNCTIONS APPLIED IN GEOSCIENCES AND ENGINEERING COMPUTATIONS
}

In applications of mathematics involving either the Laplace or the Helmholtz equation in spherical coordinates the associated Legendre equation occurs. Its solutions are called associated Legendre functions. They have some relations to classical Legendre orthogonal polynomials and classical Jacobi orthogonal polynomials. The classical Legendre polynomials and the associated Legendre functions have been used by some authors at the approximation of length of curves and cylindrical surfaces and the approximation of Earth shape in geosciences and engineering computations. In the present paper we investigate some relations between these functions pointing out possible applications in geosciences.

\section{Definition and basic properties of orthogonal polynomials}

Definition 1. Let $\langle a, b\rangle \subset R$ be a finite or infinite interval. A function $v(x)$ is called the weight function if at this interval it fulfills the following conditions:

(i) $v(x)$ is nonnegative at $\langle a, b\rangle$, i.e.

$v(x) \geq 0$

(ii) $v(x)$ is integrable at $\langle a, b\rangle$, i.e.

$0<\int_{a}^{b} v(x) d x<\infty$

and

(iii) for every $n=1,2,3 \ldots$

$0<\int_{a}^{b}|x|^{n} v(x) d x<\infty$.

Definition 2. Let $\left\{P_{n}(x)\right\}_{n=0}^{\infty}$ be a system of polynomials, where every polynomial $P_{n}(x)$ has the degree $n$. If for all polynomials of this system

$$
\int_{a}^{b} P_{n}(x) P_{m}(x) v(x) d x=0, n \neq m,
$$

then the polynomials $\left\{P_{n}(x)\right\}_{n=0}^{\infty}$ are called orthogonal in $\langle a, b\rangle$ with respect to the weight function $v(x)$. If moreover for every $n=1,2,3 \ldots$

$$
\left\|P_{n}(x)\right\|_{v(x)}=\left[\int_{a}^{b} P_{n}^{2}(x) v(x) d x\right]^{\frac{1}{2}}=1,
$$

then the polynomials are called orthonormal in $\langle a, b\rangle$ with respect to $v(x)$.

Remark 1. The condition of the orthonormality of the system $\left\{P_{n}(x)\right\}_{n=0}^{\infty}$ has the form

$$
\int_{a}^{b} P_{n}(x) P_{m}(x) v(x) d x=\delta_{n m},
$$

where $\delta_{n m}=1$ for $n=m$ and $\delta_{n m}=0$ for $n \neq m$.

Theorem 1. For every weight function $v(x)$ there exists one and only one system of polynomials $\left\{P_{n}(x)\right\}_{n=0}^{\infty}$ orthonormal in $\langle a, b\rangle$, where

$$
P_{n}(x)=\sum_{k=0}^{n} a_{k}^{(n)} x^{n-k} ; \quad a_{0}^{(n)}>0 .
$$

Proof. E. g. in [12].

Theorem 2. A polynomial $P_{n}(x)$ is orthogonal in $\langle a, b\rangle$ with respect to the weight function $v(x)$, if and only if for arbitrary polynomial $S_{m}(x)$ of the degree $m<n$ the following condition is fulfilled

$$
\int_{a}^{b} P_{n}(x) S_{m}(x) v(x) d x=0 .
$$

Proof. E. g. in [12].

Theorem 3. If the interval of orthogonality is symmetric according to the origin of coordinate system and a weight function $v(x)$ is even function, then every orthogonal polynomial $P_{n}(x)$ fulfils the equality

$$
P_{n}(-x)=(-1)^{n} P_{n}(x)
$$

\footnotetext{
* Vladimir Guldan, Mariana Marcokova

Department of Mathematics, Faculty of Science, University of Zilina, Slovakia, E-mail: vladimir.guldan@post.sk, mariana.marcokova@fpv.uniza.sk
} 
Proof. E. g. in [12].

\section{Jacobi polynomials, Legendre polynomials, Legendre associated functions}

It is well-known that Jacobi polynomials $\left\{P_{n}(x ; \alpha, \beta)\right\}_{n=0}^{\infty}$ are orthogonal in the interval $I=\langle-1,1\rangle$ with respect to the weight function

$$
J(x)=(1-x)^{\alpha}(1+x)^{\beta}, x \in(-1,1),
$$

where $\alpha>-1, \beta>-1$. Very important special case of Jacobi polynomials are classical Legendre polynomials $\left\{P_{n}(x ; 0,0)\right\}_{n=0}^{\infty}$, for which $\alpha=\beta=0$ in the weight function $J(x)$. In the next we denote them by $\left\{P_{n}(x)\right\}_{n=0}^{\infty}$.

Classical orthogonal polynomials are solutions of the second order linear homogeneous differential equations of the form (cf. e.g. [12])

$$
a(x) y_{n}^{\prime}(x)+b(x) y_{n}^{\prime \prime}(x)+\lambda_{n} y_{n}(x)=0,
$$

where $a(x)$ is a polynomial of the degree at most $2, b(x)$ is the polynomial of the degree 1 and $\lambda_{n}$ does not depend of $x$. For the classical Jacobi polynomials this equation has the form

$$
\begin{aligned}
& \left(1-x^{2}\right) y_{n}^{\prime \prime}(x)+[\beta-\alpha-(\alpha+\beta+2) x] y_{n}^{\prime}(x)+ \\
& +n(n+\alpha+\beta+1) y_{n}(x)=0,
\end{aligned}
$$

which in the case of classical Legendre polynomials is reduced to the form

$$
\left(1-x^{2}\right) y_{n}^{\prime \prime}(x)-2 x y_{n}^{\prime}(x)+n(n+1) y_{n}(x)=0 .
$$

Associated Legendre equation occurs in applications characterized by Laplace or Helmholtz equation in spherical coordinates For $m=0,1,2, \ldots n$ it has the form

$$
\left(1-x^{2}\right) y_{n}^{\prime \prime}(x)-2 x y_{n}^{\prime}(x)+\left[n(n+1)-\frac{m^{2}}{1-x^{2}}\right] y_{n}(x)=0 .
$$

Observe that for $m=0$ it reduces to Legendre equation (2). Its solutions are called associated Legendre functions of the first and second kind, respectively. They are defined by (cf. [1])

$$
P_{n}^{m}(x)=\left(1-x^{2}\right)^{\frac{m}{2}} \frac{d^{m}}{d x^{m}} P_{n}(x)
$$

and

$$
R_{n}^{m}(x)=\left(1-x^{2}\right)^{\frac{m}{2}} \frac{d^{m}}{d x^{m}} R_{n}(x),
$$

respectively. Here $P_{n}(x)$ and $R_{n}(x)$ are solutions of the Legendre equation (2), the first of them are the classical Legendre polynomials.

\section{Classical Legendre polynomials and generalized Legendre polynomials}

As it is seen from preliminaries, the Legendre classical polynomials $\left\{P_{n}(x)\right\}_{n=0}^{\infty}$ are orthogonal in $I=\langle-1,1\rangle$ with respect to the weight function $L(x)=1$.

Now we introduce the system of polynomials $\left\{Q_{n}(x)\right\}_{n=0}^{\infty}$ which will be the polynomials orthonormal in $I$ with respect to the weight function

$$
Q(x)=\left(x^{2}\right)^{\gamma},
$$

where $\gamma>0$ and $Q_{n}(+\infty)>0$. It is clear that these polynomials are generalization of the classical Legendre polynomials, which can be obtained by substituting $\gamma=0$ in the weight function $Q(x)$.

Further, we introduce two classes of orthonormal polynomials:

(a) polynomials $\left\{P_{n}\left(x ; 0, \gamma-\frac{1}{2}\right)\right\}_{n=0}^{\infty}$ orthonormal in $I$ with respect to the weight function

$$
J_{1}(x)=(1+x)^{y-\frac{1}{2}}
$$

and

(b) polynomials $\left\{P_{n}(x ; 0, \gamma)\right\}_{n=0}^{\infty}$ orthonormal in $I$ with respect to the weight function

$$
J_{2}(x)=(1+x)^{\gamma} .
$$

In both these cases we have classical Jacobi polynomials orthogonal with the weight function (1) for $\alpha=0, \beta=\gamma-1 / 2$ and $\alpha=0, \beta=\gamma$, respectively. In the next we establish relations between them and the polynomials $\left\{Q_{n}(x)\right\}_{n=0}^{\infty}$.

\section{Jacobi and Legendre classical polynomials with different weight functions}

Theorem 4. In the notations introduced in the previous sections

$$
Q_{2 n}(x)=2^{\frac{\gamma}{2}-\frac{1}{4}} P_{n}\left(2 x^{2}-1 ; 0, \gamma-\frac{1}{2}\right)
$$

and

$$
Q_{2 n+1}(x)=2^{\frac{\gamma}{2}} x P_{n}\left(2 x^{2}-1 ; 0, \gamma\right) .
$$

Proof. According to the Theorem 3, the function $Q_{2 n}(x)$ is even function. Putting $t=x^{2}$ we denote $W_{n}(t)=Q_{2 n}(x)$. The orthogonality of the polynomials $\left\{Q_{n}(x)\right\}_{n=0}^{\infty}$ for $r=0,1, \ldots, \mathrm{n}-1$ and $n>0$ yields 


$$
\begin{aligned}
& 0=\int_{0}^{1} x^{2 r} Q_{2 n}(x) x^{2 \gamma} d x=\frac{1}{2} \int_{0}^{1} t^{r} W_{n}(t) t^{\gamma-\frac{1}{2}} d t= \\
& =\frac{1}{2^{2}} \int_{-1}^{1}\left(\frac{\tau+1}{2}\right)^{r} W_{n}\left(\frac{\tau+1}{2}\right)\left(\frac{\tau+1}{2}\right)^{\gamma-\frac{1}{2}} d \tau= \\
& =\frac{1}{2^{\gamma+\frac{3}{2}}} \int_{-1}^{1}\left(\frac{\tau+1}{2}\right)^{r} W_{n}\left(\frac{\tau+1}{2}\right)(\tau+1)^{\gamma-\frac{1}{2}} d \tau .
\end{aligned}
$$

From that it is clear that the polynomials $W_{n}\left(\frac{x+1}{2}\right)$ are orthogonal in $I$ with respect to the weight function $J_{1}(x)$. According to the Theorem 1, taking into account the uniqueness of these polynomials, we have

$$
W_{n}\left(\frac{x+1}{2}\right)=k P_{n}\left(x ; 0, \gamma-\frac{1}{2}\right),
$$
where $k>0$ in consequence of the fact that $P_{n}\left(\infty ; 0, \gamma-\frac{1}{2}\right)>0$
and $W_{n}(+\infty)>0$.

From the orthonormality of the polynomials $W_{n}(t)$ we derive

$$
\begin{aligned}
& \frac{1}{2}=\int_{0}^{1} W_{n}^{2}(t) t^{\gamma-\frac{1}{2}} d t=k^{2} \int_{-1}^{1} P_{n}^{2}\left(\tau ; 0, \gamma-\frac{1}{2}\right)\left(\frac{\tau+1}{2}\right)^{\gamma-\frac{1}{2}} \frac{1}{2} d \tau= \\
& =\frac{1}{2^{\gamma+\frac{1}{2}}} k^{2} \int_{-1}^{1} P_{n}^{2}\left(\tau ; 0, \gamma-\frac{1}{2}\right)(\tau+1)^{\gamma-\frac{1}{2}} d \tau
\end{aligned}
$$

from where we have $k=2_{2}^{\frac{\gamma}{2}-\frac{1}{4}}$ and the relation (4), i.e.

$$
Q_{2 n}(x)=2^{\frac{\gamma}{2}-\frac{1}{4}} P_{n}\left(2 t-1 ; 0, \gamma-\frac{1}{2}\right), \quad t=x^{2} .
$$

Now we prove the relation (5). Putting $t=x^{2}$ we have

$$
\bar{W}_{n}(t)=x^{-1} Q_{2 n+1}(x),
$$

where $\bar{W}_{n}(t)$ is the polynomial of the degree $n$ and $Q_{2 n+1}(x)$ is odd function. For $r=0,1, \ldots, n-1$ and $n>0$ the orthogonality of the polynomials $\left\{Q_{n}(x)\right\}_{n=0}^{\infty}$ yields

$$
\begin{aligned}
& 0=\int_{0}^{1} x^{2 r+1} Q_{2 n+1}(x) x^{2 \gamma} d x=\frac{1}{2} \int_{0}^{1} t^{r} \bar{W}_{n}(t) t^{\gamma+\frac{1}{2}} d t= \\
& =\frac{1}{2^{2}} \int_{-1}^{1}\left(\frac{\tau+1}{2}\right)^{r} \bar{W}_{n}\left(\frac{\tau+1}{2}\right)\left(\frac{\tau+1}{2}\right)^{\gamma+\frac{1}{2}} d \tau= \\
& =\frac{1}{2^{\gamma+\frac{5}{2}}} \int_{-1}^{1}\left(\frac{\tau+1}{2}\right)^{r}\left(\frac{\tau+1}{2}\right)^{\frac{1}{2}} \bar{W}_{n}\left(\frac{\tau+1}{2}\right)(\tau+1)^{\gamma} d \tau .
\end{aligned}
$$

\section{From there}

$$
\left(\frac{x+1}{2}\right)^{\frac{1}{2}} \bar{W}_{n}\left(\frac{x+1}{2}\right)=\bar{k} P_{n}(x ; 0, \gamma),
$$

where $\bar{k}>0$ and from the orthonormality of the polynomials $t_{2}^{\frac{1}{2}} \bar{W}_{n}(t)$ we derive

$$
\begin{aligned}
& \frac{1}{2}=\int_{0}^{1} x^{-2} Q_{2 n+1}^{2}(x) x^{2 \gamma} d x=\int_{0}^{1} t \bar{W}_{n}^{2}(t) t^{\gamma} d t= \\
& =\frac{1}{2} \int_{-1}^{1}\left(\frac{\tau+1}{2}\right) \bar{W}_{n}^{2}\left(\frac{\tau+1}{2}\right)\left(\frac{\tau+1}{2}\right)^{\gamma} d \tau= \\
& =\frac{1}{2^{\gamma+1}} \bar{k}^{2} \int_{-1}^{1} P_{n}^{2}(\tau ; 0, \gamma)(\tau+1)^{\gamma} d \tau .
\end{aligned}
$$

Finally we get $\bar{k}=2_{2}^{\frac{\gamma}{2}}$ and the relation (5) of the theorem.

\section{Applications in geosciences}

Classical Jacobi orthogonal polynomials and their special cases - Legendre polynomials as well as their generalizations and associated Legendre functions are important tools in investigating geoscientific problems. Such geosciences like geodesy, geography, geology, geophysics, meteorology and others need adequate mathematical tools called geomathematics (cf. [3, 9]). We point out some problems of geomathematics where the functions investigated in previous sections of this paper are used.

In [5] we suggested to compute the length of a junction curve of a railroad between two sections of a rail with different curvatures by means of classical Legendre polynomials. Junction curve is a curve inserted between a straight line and a circle arc. Junction curve enables fluent transition between two sections of a rail with different curvatures. At our railroads a cubic parabola is used as the junction curve. In [2] the length of such junction curve is computed by using binomial series, because the integral of the function

$$
\sqrt{1+\left[f^{\prime}(x)\right]^{2}}, f(x)=c x^{3},
$$

(is the specific constant) had to be computed. We suggested to use Legendre polynomials, because the best approximation of a function by means of a polynomial is the approximation by means of an expansion of the function into a series of orthogonal polynomials followed by substituting the function by the partial sum of such series (cf. [12]). So we used the following expansion of a function $\sqrt{1+x}$ (cf. [12])

$$
\sqrt{1+x}=\frac{4}{3 \sqrt{2}} P_{0}(x)-\frac{4}{\sqrt{2}} \sum_{n=1}^{\infty} \frac{(-1)^{n} P_{n}(x)}{(2 n-1)(2 n+3)},
$$

where $\left\{P_{n}(x)\right\}_{n=0}^{\infty}$ are classical Legendre polynomials.

Another application of classical Legendre polynomials can be found in [6-7]. The authors used Legendre polynomials for the approximation of cylindrical surfaces which was submitted as a strategy of measurement supported by experimental verification. The results were very good - the precision was high.

Legendre associated functions were used in [14-15] for the approximation of the Earth shape. The author uses the functions $P_{n}^{m}(\sin \varphi)(\varphi$ is geocentric coordinate of geocentric radiusvector of a point of the Earth) defined by (3). The gravity potential of the 
Earth is expressed by expansion into the series of spherical functions, where the functions $P_{n}^{m}(\sin \varphi)$ occur.

Geomathematics uses also expansions of functions in two variables into double series of orthogonal polynomials ( $\mathrm{cf}$. [8, 13]), orthogonal transforms (cf. [10-11]) and wavelets theory (cf. [16]).

\section{Conclusion}

In this paper we proved relations (4) and (5) which may be used as expressions of the classical Legendre polynomials of the argument $2 x^{2}-1$ by means of generalized Legendre polynomials $\left\{Q_{n}(x)\right\}_{n=0}^{\infty}$ of the argument $x$. Other relations for the orthogonal polynomials in general in the forms of recurrence relations can be found in [4]. The Legendre associated functions connected with the above polynomials are the important part of geomathematics - mathematics developed for solutions of geoscientific problems.

Acknowledgment: This research has been supported by the Slovak Grant Agency VEGA through the project No. 1/0867/08.

\section{References}

[1] ANDREWS, L.C.: Special functions of mathematics for engineers, McGraw - Hill, Inc. 1992.

[2] BITTERER, L.: Geometry of rail (in Slovak), University of Zilina 1997.

[3] FREEDEN, W., GERVENS, T., SCHREINER, M.: Constructive Approximation on the Sphere. With Applications to Geomathematics, Oxford: Clarendon Press 1998.

[4] FTOREK, B.: Differential Recurrence Formula for the Second Derivatives of Orthogonal Polynomials, Studies of University in Zilina - math.-phys. series, Vol. 13(2001), 75-79.

[5] GULDAN, V.: Infinite Series, Special Functions and Study Programme Geodesy and Cartography (in Slovak), 3rd Zilina's didactic conference DIDZA 2006 (2006), 5 p. on CD.

[6] JANECKI, D., STEPIEN, K.: Legendre Polynomials Used for the Approximation of Cylindrical Surfaces, Communications Scientific Letters of the University of Zilina, 4/2005, 59-61.

[7] JANECKI, D., STEPIEN, K.: Legendre Polynomials Used for the Approximation of Cylindrical Surfaces, Proc. of International Conference Transcom 2005, Section 6, Zilina 2005 (2005), 269-272.

[8] KOVACIK, O.: Once More on the Double Fourier-Haar Series, Mathematica Slovaca 52 (2002), 215-220.

[9] KRCHO, J.: Modelling of Georelief and its Geometrical Structure Using DTM: Positional and Numerical Accuracy, Q111 Publishers 2001

[10] MAMRILLA, D.: On the Systems of First Order Quasi-linear Differential Equations, Tribun EU 2008.

[11] MARCOKOVA, M., GULDAN, V.: On one Orthogonal Transform Applied on a System of Orthogonal Polynomials in Two Variables, Aplimat: Journal of Applied Mathematics, Vol.2., No.2 (2009), 239-245.

[12] SUJETIN, P. K.: Classical Orthogonal Polynomials (in Russian), Nauka Moskva 1979.

[13] SUJETIN, P. K.: Orthogonal Polynomials in Two Variables, Gordon and Breach Science Publishers 1999.

[14] TENZER, R.: Geopotential Model of Earth - Approximation of Earth Shape, Geopotential Model Testing Methods, Communications - Scientific Letters of the University of Zilina, 4/2001, 50-58.

[15] TENZER, R.: Methodology for Testing of Geopotential Model, Studies of University of Zilina - Civil Engineering series (2002), 113-114.

[16] WALTER, G. G.: Wavelets and Other Orthogonal Systems with Applications, CRC Press 1994. 